ESAIM: COCV 20 (2014) 823-839

DOI: $10.1051 / \mathrm{cocv} / 2013085$
ESAIM: Control, Optimisation and Calculus of Variations

www.esaim-cocv.org

\title{
GLOBAL CARLEMAN ESTIMATE FOR STOCHASTIC PARABOLIC EQUATIONS, AND ITS APPLICATION*
}

\author{
$\mathrm{XU} \mathrm{LIU}^{1}$
}

\begin{abstract}
This paper is addressed to proving a new Carleman estimate for stochastic parabolic equations. Compared to the existing Carleman estimate in this respect (see [S. Tang and X. Zhang, SIAM J. Control Optim. 48 (2009) 2191-2216.], Thm. 5.2), one extra gradient term involving in that estimate is eliminated. Also, our improved Carleman estimate is established by virtue of the known Carleman estimate for deterministic parabolic equations. As its application, we prove the existence of insensitizing controls for backward stochastic parabolic equations. As usual, this insensitizing control problem can be reduced to a partial controllability problem for a suitable cascade system governed by a backward and a forward stochastic parabolic equation. In order to solve the latter controllability problem, we need to use our improved Carleman estimate to establish a suitable observability inequality for some linear cascade stochastic parabolic system, while the known Carleman estimate for forward stochastic parabolic equations seems not enough to derive the desired inequality.
\end{abstract}

Mathematics Subject Classification. 93B05, 93B07.

Received March 6, 2013. Revised September 4, 2013.

Published online June 5, 2014.

\section{INTRODUCTION AND MAIN RESULTS}

Carleman-type estimates are one of important tools to study partial differential equations and the related inverse/control problems. For example, the local Carleman estimate for elliptic operators with two independent variables was introduced in [4] for studying the uniqueness problem. Later, more general results on uniqueness problems for partial differential operators were given in $[3,7,30]$, respectively, by virtue of the Carleman estimate method. Also, the unique continuation properties of some evolution equations were derived by the local Carleman estimates in [22]. Moreover, global Carleman estimates for general linear parabolic equations with homogeneous boundary conditions and smooth diffusion coefficients were obtained in [6,9], respectively. They have been extensively used to establish observability inequalities in controllability problems and stability results for inverse problems. In order to obtain some estimate on the pressure in linearized Navier-Stokes equations in the context of controllability problems, a global Carleman estimate for elliptic operators with nonhomogeneous Dirichlet boundary conditions was established in [8]. Recently, some Carleman estimates for parabolic operators with

Keywords and phrases. Carleman estimate, stochastic parabolic equation, insensitizing control, controllability.

* This work is partially supported by the NSF of China under grants 11371084 and 11171060, by the National Basic Research Program of China (973 Program) under grant 2011CB808002, and by Program for New Century Excellent Talents in University under grant NCET-12-0812.

1 School of Mathematics and Statistics, Northeast Normal University, Changchun 130024, P.R. China. liuxu@amss.ac.cn 
anisotropic diffusion coefficients having jumps at interfaces were established in [18], which can be used to study the controllability of some parabolic equations with discontinuous diffusion coefficients. Nevertheless, to the best of our knowledge, very little is known about Carleman estimates for stochastic parabolic equations. In this respect, we refer to [17,23] for some known results. In [17], a global Carleman estimate for linear forward stochastic parabolic equations was established and based on this estimate, some inverse problems for stochastic parabolic equations were studied. In [23], in order to establish the controllability for general linear forward/backward stochastic parabolic equations, suitable global Carleman estimates for backward/forward stochastic parabolic equations were derived. Notice that in $[17,23]$, a gradient term (with respect to diffusion terms of forward stochastic parabolic equations) appears on the resulting Carleman estimates. This leads to more requirement on the regularity for coefficients in studying the controllability and inverse problems for stochastic parabolic equations. Furthermore, these known Carleman estimates cannot be used to establish the controllability for some coupled stochastic parabolic systems (see Rem. 5.2 for more explanations). The main purposes of this paper are to prove a new Carleman estimate for forwards stochastic parabolic equations, and give its application in the insensitizing control problem for backward stochastic parabolic equations.

To begin with, we introduce some notations. Let $G$ be a bounded domain in $\mathbb{R}^{n}(n \in \mathbb{N} \backslash\{0\})$ with a boundary $\Gamma$ of class $C^{4}$ and $T>0$. Put $Q=G \times(0, T)$ and $\Sigma=\Gamma \times(0, T)$. Assume $G_{0}, \mathcal{O}_{1}$ and $\mathcal{O}_{2}$ to be three given nonempty open subsets of $G$ such that $G_{0} \cap \mathcal{O}_{1} \neq \emptyset$. Denote by $\chi_{G_{0}}$ the characteristic function of the set $G_{0}$. Fix a complete filtered probability space $\left(\Omega, \mathcal{F},\left\{\mathcal{F}_{t}\right\}_{t \geq 0}, \mathcal{P}\right)$, on which a one-dimensional standard Brownian motion $\{w(t)\}_{t \geq 0}$ is defined such that $\left\{\mathcal{F}_{t}\right\}_{t \geq 0}$ is the natural filtration generated by $w(\cdot)$, augmented by all the $\mathcal{P}$-null sets in $\mathcal{F}$. Let $\mathcal{H}$ be a Banach space, and let $C([0, T] ; \mathcal{H})$ be the Banach space of all $\mathcal{H}$-valued strongly continuous abstract functions defined on $[0, T]$. We denote by $L_{\mathcal{F}}^{2}(0, T ; \mathcal{H})$ the Banach space consisting

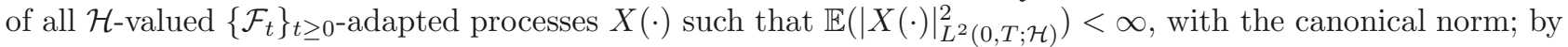
$L_{\mathcal{F}}^{\infty}(0, T ; \mathcal{H})$ the Banach space consisting of all $\mathcal{H}$-valued $\left\{\mathcal{F}_{t}\right\}_{t \geq 0}$-adapted essentially bounded processes; and by $L_{\mathcal{F}}^{2}(\Omega ; C([0, T] ; \mathcal{H}))$ the Banach space consisting of all $\mathcal{H}$-valued $\left\{\mathcal{F}_{t}\right\}_{t \geq 0}$-adapted continuous processes $X(\cdot)$ such that $\mathbb{E}\left(|X(\cdot)|_{C([0, T] ; \mathcal{H})}^{2}\right)<\infty$. Similarly, one can define $L_{\mathcal{F}}^{\infty}\left(\Omega ; C^{m}([0, T] ; \mathcal{H})\right)$ for any positive integer $m$. Moreover, it is well known that (see e.g. [6]), there exists a function $\psi \in C^{4}(\bar{G})$ such that

$$
\psi(x)>0, \text { in } G ; \quad \psi(x)=0, \text { on } \Gamma ; \quad|\nabla \psi(x)|>0, \text { in } \overline{G \backslash G_{1}},
$$

where $G_{1}$ is any given nonempty open subset of $G$ such that $\overline{G_{1}} \subseteq G_{0} \cap \mathcal{O}_{1}$. For any fixed $k \geq 1$, and positive parameters $\beta$ and $\lambda$, write

$$
\gamma(t)=\frac{1}{t^{k}(T-t)^{k}}, \quad \varphi(x, t)=\frac{\mathrm{e}^{\beta \psi(x)}}{t^{k}(T-t)^{k}}, \quad \alpha(x, t)=\frac{\mathrm{e}^{\beta \psi(x)}-\mathrm{e}^{2 \beta|\psi|_{C(\bar{G})}}}{t^{k}(T-t)^{k}}, \quad \theta=\mathrm{e}^{\lambda \alpha} .
$$

Furthermore, we assume that the coefficients $a^{i j}: \Omega \times \bar{G} \times[0, T] \rightarrow \mathbb{R}(i, j=1,2, \ldots, n)$ satisfy the following conditions:

(1) $a^{i j} \in L_{\mathcal{F}}^{\infty}\left(\Omega ; C^{1}\left([0, T] ; W^{1, \infty}(G)\right)\right)$ and $a^{i j}=a^{j i}$;

(2) there is some positive constant $\rho_{0}$ such that

$$
\sum_{i, j=1}^{n} a^{i j}(\omega, x, t) \varsigma^{i} \varsigma^{j} \geq \rho_{0}|\varsigma|^{2} \quad \text { for any }(\omega, x, t, \varsigma)=\left(\omega, x, t, \varsigma^{1}, \ldots, \varsigma^{n}\right) \in \Omega \times Q \times \mathbb{R}^{n} .
$$

Set $A=\sup _{1 \leq i, j \leq n}\left|a^{i j}\right|_{L_{\mathcal{F}}^{\infty}\left(\Omega ; C^{1}\left([0, T] ; W^{1, \infty}(G)\right)\right)}$.

Consider the following linear forward stochastic parabolic equation:

$$
\begin{cases}\mathrm{d} h-\sum_{i, j=1}^{n}\left(a^{i j} h_{x_{i}}\right)_{x_{j}} \mathrm{~d} t=F_{1} \mathrm{~d} t+F_{2} \mathrm{~d} w(t) & \text { in } Q, \\ h=0 & \text { on } \Sigma, \\ h(0)=h_{0} & \text { in } G,\end{cases}
$$


where $F_{i} \in L_{\mathcal{F}}^{2}\left(0, T ; L^{2}(G)\right)(i=1,2)$ and $h_{0} \in L^{2}\left(\Omega, \mathcal{F}_{0}, \mathcal{P} ; L^{2}(G)\right)$. By [10], it is easy to check that $(1.2)$ admits one and only one solution $h$ in the class of

$$
h \in L_{\mathcal{F}}^{2}\left(\Omega ; C\left([0, T] ; L^{2}(G)\right)\right) \bigcap L_{\mathcal{F}}^{2}\left(0, T ; H_{0}^{1}(G)\right) .
$$

The first main result of this paper is the following global Carleman estimate for (1.2):

Theorem 1.1. There exists a positive constant $\beta_{1}$, depending only on $n, G, G_{1}, T, \rho_{0}$ and $A$, such that for any $\beta \geq \beta_{1}$, one can find two positive constants $\lambda_{1}=\lambda_{1}(\beta)$ and $C=C(\beta)$ so that for any $h_{0} \in L^{2}\left(\Omega, \mathcal{F}_{0}, \mathcal{P} ; L^{2}(G)\right)$ and $F_{i} \in L_{\mathcal{F}}^{2}\left(0, T ; L^{2}(G)\right)(i=1,2)$, the corresponding solution $h$ of $(1.2)$ satisfies

$$
\mathbb{E} \int_{Q} \theta^{2}\left(\lambda^{3} \gamma^{3} h^{2}+\lambda \gamma|\nabla h|^{2}\right) \mathrm{d} x \mathrm{~d} t \leq C\left(\mathbb{E} \int_{0}^{T} \int_{G_{1}} \theta^{2} \lambda^{3} \gamma^{3} h^{2} \mathrm{~d} x \mathrm{~d} t+\mathbb{E} \int_{Q} \theta^{2} F_{1}^{2} \mathrm{~d} x \mathrm{~d} t+\mathbb{E} \int_{Q} \theta^{2} \lambda^{2} \gamma^{2} F_{2}^{2} \mathrm{~d} x \mathrm{~d} t\right),
$$

for any $\lambda \geq \lambda_{1}$.

Remark 1.2. Compared to the known Carleman estimate for forward stochastic parabolic equations (see [23], Thm. 5.2), we eliminate an extra term involving $\nabla F_{2}$. Meanwhile, in the weighted functions $\gamma$ and $\theta$, the value of the parameter $k$ is allowed to be only larger than or equal to 1 rather than 2 . Moreover, the coefficients $a^{i j}(i, j=1,2, \ldots, n)$ are only $W^{1, \infty}$ functions with respect to the space variable rather than $W^{2, \infty}$ ones. Therefore, using this new Carleman estimate, we can relax some assumption on the regularity for coefficients of stochastic parabolic equations in the context of the controllability and inverse problems.

Remark 1.3. Carleman-type estimates are a kind of weighted energy estimates. For example, suppose that in (1.2), $F_{1}=F_{2}=0$ in $Q, \mathcal{P}$-a.s. Roughly speaking, the Carleman estimate (1.3) means that the local information (in the domain $G_{1}$ ) of the solution $h$ for equation (1.2) can determine the whole one of it.

Remark 1.4. By a similar method used in the proof of Theorem 1.1, it is easy to show the following global Carleman estimate for (1.2) in $H^{-1}$-space. There exists a positive constant $\beta_{2}$, depending only on $n, G, G_{1}, T, \rho_{0}$ and $A$, such that for any $\beta \geq \beta_{2}$, one can find two positive constants $\lambda_{2}=\lambda_{2}(\beta)$ and $C=C(\beta)$ so that for any $F_{1} \in L_{\mathcal{F}}^{2}\left(0, T ; H^{-1}(G)\right), F_{2} \in L_{\mathcal{F}}^{2}\left(0, T ; L^{2}(G)\right)$ and $h_{0} \in L^{2}\left(\Omega, \mathcal{F}_{0}, \mathcal{P} ; L^{2}(G)\right)$, the corresponding solution $h$ of (1.2) satisfies

$\mathbb{E} \int_{Q} \theta^{2}\left(\lambda \gamma^{3} h^{2}+\lambda^{-1} \gamma|\nabla h|^{2}\right) \mathrm{d} x \mathrm{~d} t \leq C\left(\mathbb{E} \int_{0}^{T} \int_{G_{1}} \theta^{2} \lambda \gamma^{3} h^{2} \mathrm{~d} x \mathrm{~d} t+\mathbb{E} \int_{0}^{T} \gamma^{2}\left|\theta F_{1}\right|_{H^{-1}(G)}^{2} \mathrm{~d} t+\mathbb{E} \int_{Q} \theta^{2} \gamma^{2} F_{2}^{2} \mathrm{~d} x \mathrm{~d} t\right)$,

for any $\lambda \geq \lambda_{2}$.

The Carleman estimate for forward stochastic parabolic equations in [23] was established by virtue of a weighted energy identity for equations themselves. However, in order to get the improved Carleman estimates (1.3) and (1.4), we adopt a different new method by establishing some relationship between Carleman estimates for forward stochastic parabolic equations and those for deterministic parabolic equations. More precisely, we first transform the desired Carleman estimates for forward stochastic parabolic equations into a null controllability problem for a certain backward stochastic parabolic equation. Then, this controllability problem is reduced to a Carleman estimate for some forward random parabolic equation. Therefore, by this approach, we can establish a new Carleman estimate for forward stochastic parabolic equations by virtue of the known Carleman estimates for deterministic parabolic equations directly. Notice that, as a byproduct, this new Carleman estimate can be used to improve the known controllability result for backward stochastic parabolic equations by relaxing some assumption on the coefficients (see Rem. 2.3 for more explanations). 
As a typical application of the Carleman estimate (1.3), we study the insensitivity problems for the following controlled backward stochastic parabolic equation:

$$
\begin{cases}\mathrm{d} y+\Delta y \mathrm{~d} t=\left(\xi+\mu Y+\chi_{G_{0}} u\right) \mathrm{d} t+(Y+v) \mathrm{d} w(t) & \text { in } Q, \\ y=0 & \text { on } \Sigma, \\ y(T)=y_{T}+\tau \widehat{y}_{T} & \text { in } G,\end{cases}
$$

where $(u, v)$ is the control variable, $(y, Y)$ is the state variable, $\mu \in L_{\mathcal{F}}^{\infty}\left(0, T ; L^{\infty}(G)\right)$ is a given process, $\xi \in$ $L_{\mathcal{F}}^{2}\left(0, T ; L^{2}(G)\right)$ and $y_{T} \in L^{2}\left(\Omega, \mathcal{F}_{T}, \mathcal{P} ; L^{2}(G)\right)$ are, respectively, a known heat source and a given terminal state, $\tau$ is an unknown small real number, and $\widehat{y}_{T} \in L^{2}\left(\Omega, \mathcal{F}_{T}, \mathcal{P} ; L^{2}(G)\right)$ is unknown. By the duality analysis as that in [27], one can prove that for any $(u, v) \in L_{\mathcal{F}}^{2}\left(0, T ; L^{2}\left(G_{0}\right)\right) \times L_{\mathcal{F}}^{2}\left(0, T ; L^{2}(G)\right), \tau \in \mathbb{R}$ and $\widehat{y}_{T} \in$ $L^{2}\left(\Omega, \mathcal{F}_{T}, \mathcal{P} ; L^{2}(G)\right),(1.5)$ admits one and only one solution in the class of

$$
(y, Y) \in\left(L_{\mathcal{F}}^{2}\left(\Omega ; C\left([0, T] ; L^{2}(G)\right)\right) \bigcap L_{\mathcal{F}}^{2}\left(0, T ; H_{0}^{1}(G)\right)\right) \times L_{\mathcal{F}}^{2}\left(0, T ; L^{2}(G)\right) .
$$

Define the following energy functional for (1.5):

$$
\Phi(y, Y)=\frac{1}{2} \mathbb{E} \int_{0}^{T} \int_{\mathcal{O}_{1}}|y(x, t ; \tau, u, v)|^{2} \mathrm{~d} x \mathrm{~d} t+\frac{1}{2} \mathbb{E} \int_{0}^{T} \int_{\mathcal{O}_{2}}|Y(x, t ; \tau, u, v)|^{2} \mathrm{~d} x \mathrm{~d} t,
$$

where $(y, Y)=(y(x, t ; \tau, u, v), Y(x, t ; \tau, u, v))$ denotes the solution of (1.5) associated to $\tau, u$ and $v$. Introduce the following notion of insensitizing control.

Definition 1.5. For given $\xi \in L_{\mathcal{F}}^{2}\left(0, T ; L^{2}(G)\right)$ and $y_{T} \in L^{2}\left(\Omega, \mathcal{F}_{T}, \mathcal{P} ; L^{2}(G)\right)$, a pair of control functions $(u, v) \in L_{\mathcal{F}}^{2}\left(0, T ; L^{2}\left(G_{0}\right)\right) \times L_{\mathcal{F}}^{2}\left(0, T ; L^{2}(G)\right)$ is said to insensitize the functional $\Phi$ if

$$
\left.\frac{\partial \Phi(y, Y)}{\partial \tau}\right|_{\tau=0}=0, \quad \forall \widehat{y}_{T} \in L^{2}\left(\Omega, \mathcal{F}_{T}, \mathcal{P} ; L^{2}(G)\right) \text { with }\left|\widehat{y}_{T}\right|_{L^{2}\left(\Omega, \mathcal{F}_{T}, \mathcal{P} ; L^{2}(G)\right)}=1 .
$$

Backward stochastic parabolic equations have been studied in the stochastic control and nonlinear filtering theory (see $[19,21,28])$. Such equations are also useful in mathematical finance as they provide a generalized version of the celebrated Black-Scholes formula (see [20]). Roughly speaking, the insensitizing control problem of (1.5) means that we are expected to find an arbitrarily located internal controller $u$, such that the local energy $\Phi$ is almost invariant with respect to small perturbations on the terminal value $y_{T}$.

Deterministic insensitivity control problem was introduced by Lions in [12]. In [24], the author showed that when $G \backslash \overline{G_{0}} \neq \emptyset$, one could not expect the existence of insensitizing controls for every initial value $y_{0} \in L^{2}(G)$, even for the linear parabolic equation; while when $G_{0} \cap \mathcal{O}_{1} \neq \emptyset$, for $y_{0}=0$ and $\xi$ satisfying suitable assumptions, the existence of insensitizing controls was also proved in that paper for some semilinear heat equations with globally Lipschitz continuous nonlinearity and Dirichlet boundary conditions. Later, this result was extended to semilinear heat equations with superlinear nonlinearities and other boundary conditions (see e.g. [2] and the rich references therein). In [14], the existence of insensitizing controls for a class of quasilinear parabolic equations was presented.

However, very little is known for stochastic insensitivity control problems. As far as we know, there is only one published paper [25] addressing the insensitivity control problem for stochastic parabolic equations. In [25], the existence of insensitizing controls for forward stochastic heat equations was established. Also, it was commented in [25], Remark 6 that when treating the similar insensitizing control problem for backward stochastic heat equations, one might encounter the difficulty of establishing the desired observability inequality by means of known Carleman estimates. In this paper, we use the improved Carleman estimate (1.3) to overcome this difficulty. Notice however that for deterministic parabolic equations, there is no essential difference between the insensitivity problem for forward equations and that for the backward counterparts.

The other main result in this paper, stated below, is to prove the existence of insensitizing controls for the backward stochastic parabolic equation (1.5). 
Theorem 1.6. Assume that $G_{0} \cap \mathcal{O}_{1} \neq \emptyset, \mathcal{O}_{2}=G$ and $y_{T}=0$ in $G$, $\mathcal{P}$-a.s. Then there exist two positive constants $M$ and $C$ depending only on $n, T, G, G_{0}, \mathcal{O}_{1}$ and $|\mu|_{L_{\mathcal{F}}^{\infty}\left(0, T ; L^{\infty}(G)\right)}$, such that for any $\xi \in L_{\mathcal{F}}^{2}\left(0, T ; L^{2}(G)\right)$ satisfying

$$
\left|\exp \left(\frac{M}{(T-t)^{2}}\right) \xi\right|_{L_{\mathcal{F}}^{2}\left(0, T ; L^{2}(G)\right)}<\infty
$$

one can find a pair of control functions $(u, v) \in L_{\mathcal{F}}^{2}\left(0, T ; L^{2}\left(G_{0}\right)\right) \times L_{\mathcal{F}}^{2}\left(0, T ; L^{2}(G)\right)$, which insensitizes the functional $\Phi$ (defined in (1.6)) in the sense of Definition 1.5. Moreover,

$$
|u|_{L_{\mathcal{F}}^{2}\left(0, T ; L^{2}\left(G_{0}\right)\right)}+|v|_{L_{\mathcal{F}}^{2}\left(0, T ; L^{2}(G)\right)} \leq C\left|\exp \left(\frac{M}{(T-t)^{2}}\right) \xi\right|_{L_{\mathcal{F}}^{2}\left(0, T ; L^{2}(G)\right)}
$$

Several further remarks are in order.

Remark 1.7. It would be interesting to study the insensitivity problems when $\mathcal{O}_{2}$ is a nonempty subset of $G$ satisfying $\mathcal{O}_{2} \varsubsetneqq G$. However, it seems difficult to establish the desired observability estimate in this case (see Rem. 4.5).

Remark 1.8. It would be interesting to study the existence of insensitizing controls for backward stochastic parabolic equations without the extra control $v$. However, this seems to be very difficult, because in the proof of Theorem 1.6 we need to use a known Carleman estimate for backward stochastic parabolic equations [23].

The rest of this paper is organized as follows. In Section 2, we prove a new global Carleman estimate for forward stochastic parabolic equations (Thm. 1.1). In Section 3, we reduce an insensitivity control problem to a partial controllability problem of a cascade stochastic parabolic system. Section 4 is devoted to establishing a global Carleman estimate for linear cascade stochastic parabolic systems, based on the Carleman estimate (1.3). Finally, in Section 5, we give a proof of Theorem 1.6.

\section{Carleman estimate for forward stochastic parabolic Equations}

In this section, we derive a new Carleman estimate for the forward stochastic parabolic equation (1.2). Notice that when $F_{2} \equiv 0$, equation (1.2) becomes a random parabolic equation. Therefore, recalling the known Carleman estimates for deterministic parabolic equations (see e.g. $[9,15])$ and $(1.1)$, we conclude the following global Carleman estimate for random parabolic equations.

Lemma 2.1. If $F_{2} \equiv 0$, then there exist two positive constants $\beta_{0}$ and $\lambda_{0}$, depending only on $n, G, G_{1}, T, \rho_{0}$ and $A$, such that for any $F_{1} \in L_{\mathcal{F}}^{2}\left(0, T ; L^{2}(G)\right)$ and $h_{0} \in L^{2}\left(\Omega, \mathcal{F}_{0}, \mathcal{P} ; L^{2}(G)\right)$, the corresponding solution $h \in L_{\mathcal{F}}^{2}\left(0, T ; H_{0}^{1}(G)\right)$ of (1.2) (with $\left.F_{2} \equiv 0\right)$ satisfies

$$
\mathbb{E} \int_{Q} \theta^{2}\left(\lambda^{3} \gamma^{3} h^{2}+\lambda \gamma|\nabla h|^{2}\right) \mathrm{d} x \mathrm{~d} t \leq C\left(\mathbb{E} \int_{0}^{T} \int_{G_{1}} \theta^{2} \lambda^{3} \gamma^{3} h^{2} \mathrm{~d} x \mathrm{~d} t+\mathbb{E} \int_{Q} \theta^{2} F_{1}^{2} \mathrm{~d} x \mathrm{~d} t\right),
$$

for any $\beta \geq \beta_{0}$ and $\lambda \geq \lambda_{0}$.

In the following, we denote by $\beta$ and $\lambda$ two fixed constants satisfying the conditions mentioned in Lemma 2.1. Also, for convenience, in the sequel, we denote by $C$ any positive constant, depending only on $n, G, G_{0}, G_{1}, T, \rho_{0}, \beta, \mathcal{O}_{1}, A$ and $|\mu|_{L_{\mathcal{F}}^{\infty}\left(0, T ; L^{\infty}(G)\right)}$, which may be different from one place to another.

As a preliminary to prove Theorem 1.1, based on the known Carleman estimate in Lemma 2.1, we first need to study the null controllability of a backward stochastic parabolic equation by the duality technique. Indeed, for 
any fixed $F_{i} \in L_{\mathcal{F}}^{2}\left(0, T ; L^{2}(G)\right)(i=1,2)$ and $h_{0} \in L^{2}\left(\Omega, \mathcal{F}_{0}, \mathcal{P} ; L^{2}(G)\right)$, let $h$ denote the corresponding solution of equation (1.2). Consider the following controlled backward stochastic parabolic equation:

$$
\begin{cases}\mathrm{d} r+\sum_{i, j=1}^{n}\left(a^{i j} r_{x_{i}}\right)_{x_{j}} \mathrm{~d} t=\left(\theta^{2} \lambda^{3} \gamma^{3} h+\chi_{G_{1}} g\right) \mathrm{d} t+R \mathrm{~d} w(t) & \text { in } Q \\ r=0 & \text { on } \Sigma, \\ r(T)=0 & \text { in } G\end{cases}
$$

where $g$ is the control variable and $(r, R)$ is the state variable. Then, we have the following null controllability result for equation (2.1).

Proposition 2.2. There exists a control $\widehat{g} \in L_{\mathcal{F}}^{2}\left(0, T ; L^{2}\left(G_{1}\right)\right)$ such that equation (2.1) admits a solution $(\widehat{r}, \widehat{R}) \in\left(L_{\mathcal{F}}^{2}\left(\Omega ; C\left([0, T] ; L^{2}(G)\right)\right) \bigcap L_{\mathcal{F}}^{2}\left(0, T ; H_{0}^{1}(G)\right)\right) \times L_{\mathcal{F}}^{2}\left(0, T ; L^{2}(G)\right)$ associated to $\widehat{g}$ satisfying $\widehat{r}(0)=0$ in $G, \mathcal{P}$-a.s. Moreover,

$$
\mathbb{E} \int_{Q}\left(\theta^{-2} \widehat{r}^{2}+\theta^{-2} \lambda^{-3} \gamma^{-3} \widehat{g}^{2}\right) \mathrm{d} x \mathrm{~d} t+\mathbb{E} \int_{Q} \theta^{-2} \lambda^{-2} \gamma^{-2} \widehat{R}^{2} \mathrm{~d} x \mathrm{~d} t \leq C \mathbb{E} \int_{Q} \theta^{2} \lambda^{3} \gamma^{3} h^{2} \mathrm{~d} x \mathrm{~d} t
$$

Proof. We borrow some ideas from [9]. The main steps are as follows. First, we construct a family of optimal (approximate-null) control problems for equation (2.1). Next, we establish a uniform estimate for these optimal (approximate) solutions. Finally, by taking the limit, one obtains the desired null controllability result.

Step 1. For any $\varepsilon>0$, write $\alpha_{\varepsilon} \equiv \alpha_{\varepsilon}(x, t)=\frac{\mathrm{e}^{\beta \psi(x)}-\mathrm{e}^{2 \beta|\psi|_{C(\bar{G})}}}{(t+\varepsilon)^{k}(T-t+\varepsilon)^{k}}$ and construct the following optimal (approximate-null) control problem $\left(\mathbf{P}_{\varepsilon}\right)$ :

$$
\min _{g \in \mathcal{U}}\left\{\frac{1}{2} \mathbb{E} \int_{Q} \mathrm{e}^{-2 \lambda \alpha_{\varepsilon}} r^{2} \mathrm{~d} x \mathrm{~d} t+\frac{1}{2} \mathbb{E} \int_{0}^{T} \int_{G_{1}} \theta^{-2} \lambda^{-3} \gamma^{-3} g^{2} \mathrm{~d} x \mathrm{~d} t+\frac{1}{2 \varepsilon} \mathbb{E} \int_{G} r^{2}(0) \mathrm{d} x\right\},
$$

subject to equation (2.1), where $\mathcal{U}=\left\{g \in L_{\mathcal{F}}^{2}\left(0, T ; L^{2}\left(G_{1}\right)\right) ; \mathbb{E} \int_{0}^{T} \int_{G_{1}} \theta^{-2} \gamma^{-3} g^{2} \mathrm{~d} x \mathrm{~d} t<\infty\right\}$.

Similar to [11], it is easy to check that for any $\varepsilon>0$, the above optimal control problem $\left(\mathbf{P}_{\varepsilon}\right)$ admits a unique optimal solution $\left(g_{\varepsilon}, r_{\varepsilon}, R_{\varepsilon}\right) \in \mathcal{U} \times L_{\mathcal{F}}^{2}\left(0, T ; H_{0}^{1}(G)\right) \times L_{\mathcal{F}}^{2}\left(0, T ; L^{2}(G)\right)$. Moreover, by the standard variational method (see $[11,13]$ ), it follows that

$$
g_{\varepsilon}=\chi_{G_{1}} \theta^{2} \lambda^{3} \gamma^{3} z_{\varepsilon} \quad \text { in } Q, \quad \mathcal{P} \text {-a.s. }
$$

where $z_{\varepsilon}$ satisfies

$$
\begin{cases}\mathrm{d} z_{\varepsilon}-\sum_{i, j=1}^{n}\left(a^{i j} z_{\varepsilon, x_{i}}\right)_{x_{j}} \mathrm{~d} t=\mathrm{e}^{-2 \lambda \alpha_{\varepsilon}} r_{\varepsilon} \mathrm{d} t & \text { in } Q, \\ z_{\varepsilon}=0 & \text { on } \Sigma, \\ z_{\varepsilon}(0)=\frac{1}{\varepsilon} r_{\varepsilon}(0) & \text { in } G .\end{cases}
$$


Step 2. We now establish a uniform estimate for the optimal solutions $\left\{\left(g_{\varepsilon}, r_{\varepsilon}, R_{\varepsilon}\right)\right\}_{\varepsilon>0}$. By (2.1), (2.4), Itô's formula and (2.3), it follows that

$$
\begin{aligned}
& -\mathbb{E} \int_{G} r_{\varepsilon}(0) z_{\varepsilon}(0) \mathrm{d} x \\
& =\mathbb{E} \int_{Q}\left\{r_{\varepsilon}\left[\sum_{i, j=1}^{n}\left(a^{i j} z_{\varepsilon, x_{i}}\right)_{x_{j}}+\mathrm{e}^{-2 \lambda \alpha_{\varepsilon}} r_{\varepsilon}\right]+z_{\varepsilon}\left[-\sum_{i, j=1}^{n}\left(a^{i j} r_{\varepsilon, x_{i}}\right)_{x_{j}}+\theta^{2} \lambda^{3} \gamma^{3} h+\chi_{G_{1}} g_{\varepsilon}\right]\right\} \mathrm{d} x \mathrm{~d} t \\
& =\mathbb{E} \int_{Q}\left(\mathrm{e}^{-2 \lambda \alpha_{\varepsilon}} r_{\varepsilon}^{2}+\theta^{2} \lambda^{3} \gamma^{3} z_{\varepsilon} h+\chi_{G_{1}} \theta^{2} \lambda^{3} \gamma^{3} z_{\varepsilon}^{2}\right) \mathrm{d} x \mathrm{~d} t
\end{aligned}
$$

This, together with the last equality of (2.4) and Lemma 2.1, indicates that for sufficiently small $\rho>0$,

$$
\begin{aligned}
& \mathbb{E} \int_{Q}\left(\mathrm{e}^{-2 \lambda \alpha_{\varepsilon}} r_{\varepsilon}^{2}+\chi_{G_{1}} \theta^{2} \lambda^{3} \gamma^{3} z_{\varepsilon}^{2}\right) \mathrm{d} x \mathrm{~d} t+\frac{1}{\varepsilon} \mathbb{E} \int_{G} r_{\varepsilon}^{2}(0) \mathrm{d} x \\
& \leq\left|\mathbb{E} \int_{Q} \theta^{2} \lambda^{3} \gamma^{3} z_{\varepsilon} h \mathrm{~d} x \mathrm{~d} t\right| \\
& \leq \rho \mathbb{E} \int_{Q} \theta^{2} \lambda^{3} \gamma^{3} z_{\varepsilon}^{2} \mathrm{~d} x \mathrm{~d} t+C(\rho) \mathbb{E} \int_{Q} \theta^{2} \lambda^{3} \gamma^{3} h^{2} \mathrm{~d} x \mathrm{~d} t \\
& \leq \rho C\left[\mathbb{E} \int_{0}^{T} \int_{G_{1}} \theta^{2} \lambda^{3} \gamma^{3} z_{\varepsilon}^{2} \mathrm{~d} x \mathrm{~d} t+\mathbb{E} \int_{Q} \theta^{2}\left(\mathrm{e}^{-2 \lambda \alpha_{\varepsilon}} r_{\varepsilon}\right)^{2} \mathrm{~d} x \mathrm{~d} t\right]+C(\rho) \mathbb{E} \int_{Q} \theta^{2} \lambda^{3} \gamma^{3} h^{2} \mathrm{~d} x \mathrm{~d} t
\end{aligned}
$$

Notice that $\theta^{2} \mathrm{e}^{-2 \lambda \alpha_{\varepsilon}} \leq 1$. Therefore, by (2.3) and (2.5), we see that

$$
\mathbb{E} \int_{Q}\left(\mathrm{e}^{-2 \lambda \alpha_{\varepsilon}} r_{\varepsilon}^{2}+\theta^{-2} \lambda^{-3} \gamma^{-3} g_{\varepsilon}^{2}\right) \mathrm{d} x \mathrm{~d} t+\frac{1}{\varepsilon} \mathbb{E} \int_{G} r_{\varepsilon}^{2}(0) \mathrm{d} x \leq C \mathbb{E} \int_{Q} \theta^{2} \lambda^{3} \gamma^{3} h^{2} \mathrm{~d} x \mathrm{~d} t .
$$

On the other hand, by the first equation of (2.1), it follows that

$$
\begin{aligned}
d\left(\mathrm{e}^{-2 \lambda \alpha_{\varepsilon}} \lambda^{-2} \gamma^{-2} r_{\varepsilon}^{2}\right)= & \left(\mathrm{e}^{-2 \lambda \alpha_{\varepsilon}} \lambda^{-2} \gamma^{-2}\right)_{t} r_{\varepsilon}^{2} \mathrm{~d} t+\mathrm{e}^{-2 \lambda \alpha_{\varepsilon}} \lambda^{-2} \gamma^{-2}\left(\mathrm{~d} r_{\varepsilon}\right)^{2} \\
& +2 \mathrm{e}^{-2 \lambda \alpha_{\varepsilon}} \lambda^{-2} \gamma^{-2} r_{\varepsilon}\left[-\sum_{i, j=1}^{n}\left(a^{i j} r_{\varepsilon, x_{i}}\right)_{x_{j}} \mathrm{~d} t+\theta^{2} \lambda^{3} \gamma^{3} h \mathrm{~d} t+\chi_{G_{1}} g_{\varepsilon} \mathrm{d} t+R_{\varepsilon} \mathrm{d} w(t)\right]
\end{aligned}
$$

Then, by Young's inequality, this implies that

$$
\begin{aligned}
& \mathbb{E} \int_{Q} \mathrm{e}^{-2 \lambda \alpha_{\varepsilon}} \lambda^{-2} \gamma^{-2} R_{\varepsilon}^{2} \mathrm{~d} x \mathrm{~d} t+2 \mathbb{E} \int_{Q} \mathrm{e}^{-2 \lambda \alpha_{\varepsilon}} \lambda^{-2} \gamma^{-2} \sum_{i, j=1}^{n} a^{i j} r_{\varepsilon, x_{i}} r_{\varepsilon, x_{j}} \mathrm{~d} x \mathrm{~d} t \\
& =-\mathbb{E} \int_{Q}\left(\mathrm{e}^{-2 \lambda \alpha_{\varepsilon}} \lambda^{-2} \gamma^{-2}\right)_{t} r_{\varepsilon}^{2} \mathrm{~d} x \mathrm{~d} t-2 \mathbb{E} \int_{Q} \sum_{i j=1}^{n} a^{i j}\left(\mathrm{e}^{-2 \lambda \alpha_{\varepsilon}} \lambda^{-2} \gamma^{-2}\right)_{x_{j}} r_{\varepsilon, x_{i}} r_{\varepsilon} \mathrm{d} x \mathrm{~d} t \\
& -2 \mathbb{E} \int_{Q} \theta^{2} \mathrm{e}^{-2 \lambda \alpha_{\varepsilon}} \lambda \gamma r_{\varepsilon} h \mathrm{~d} x \mathrm{~d} t-2 \mathbb{E} \int_{Q} \chi_{G_{1}} \mathrm{e}^{-2 \lambda \alpha_{\varepsilon}} \lambda^{-2} \gamma^{-2} r_{\varepsilon} g_{\varepsilon} \mathrm{d} x \mathrm{~d} t .
\end{aligned}
$$


Notice that $\mathrm{e}^{-2 \lambda \alpha_{\varepsilon}} \leq \theta^{-2}$. By a simple calculation, we find that

$$
\begin{aligned}
& \mathbb{E} \int_{Q} \mathrm{e}^{-2 \lambda \alpha_{\varepsilon}} \lambda^{-2} \gamma^{-2} R_{\varepsilon}^{2} \mathrm{~d} x \mathrm{~d} t+2 \mathbb{E} \int_{Q} \mathrm{e}^{-2 \lambda \alpha_{\varepsilon}} \lambda^{-2} \gamma^{-2}\left|\nabla r_{\varepsilon}\right|^{2} \mathrm{~d} x \mathrm{~d} t \\
& \leq C\left(\mathbb{E} \int_{Q} \mathrm{e}^{-2 \lambda \alpha_{\varepsilon}} \lambda^{-1} r_{\varepsilon}^{2} \mathrm{~d} x \mathrm{~d} t+\mathbb{E} \int_{Q} \mathrm{e}^{-2 \lambda \alpha_{\varepsilon}} \lambda^{-1} \gamma^{-1}\left|\nabla r_{\varepsilon}\right|\left|r_{\varepsilon}\right| \mathrm{d} x \mathrm{~d} t\right. \\
& \left.\quad+\mathbb{E} \int_{Q} \theta \mathrm{e}^{-\lambda \alpha_{\varepsilon}} \lambda \gamma\left|r_{\varepsilon} h\right| \mathrm{d} x \mathrm{~d} t+\mathbb{E} \int_{Q} \mathrm{e}^{-2 \lambda \alpha_{\varepsilon}} \lambda^{-2} \gamma^{-2}\left|r_{\varepsilon} g_{\varepsilon}\right| \mathrm{d} x \mathrm{~d} t\right) \\
& \leq \mathbb{E} \int_{Q} \mathrm{e}^{-2 \lambda \alpha_{\varepsilon}} \lambda^{-2} \gamma^{-2}\left|\nabla r_{\varepsilon}\right|^{2} \mathrm{~d} x \mathrm{~d} t+C \mathbb{E} \int_{Q}\left(\mathrm{e}^{-2 \lambda \alpha_{\varepsilon}} r_{\varepsilon}^{2}+\mathrm{e}^{-2 \lambda \alpha_{\varepsilon}} \lambda^{-4} \gamma^{-4} g_{\varepsilon}^{2}+\theta^{2} \lambda^{3} \gamma^{3} h^{2}\right) \mathrm{d} x \mathrm{~d} t \\
& \leq \mathbb{E} \int_{Q} \mathrm{e}^{-2 \lambda \alpha_{\varepsilon}} \lambda^{-2} \gamma^{-2}\left|\nabla r_{\varepsilon}\right|^{2} \mathrm{~d} x \mathrm{~d} t+C \mathbb{E} \int_{Q}\left(\mathrm{e}^{-2 \lambda \alpha_{\varepsilon}} r_{\varepsilon}^{2}+\theta^{-2} \lambda^{-3} \gamma^{-3} g_{\varepsilon}^{2}+\theta^{2} \lambda^{3} \gamma^{3} h^{2}\right) \mathrm{d} x \mathrm{~d} t .
\end{aligned}
$$

Combining (2.7) with (2.6), we conclude that

$$
\begin{aligned}
& \mathbb{E} \int_{Q}\left(\mathrm{e}^{-2 \lambda \alpha_{\varepsilon}} r_{\varepsilon}^{2}+\theta^{-2} \lambda^{-3} \gamma^{-3} g_{\varepsilon}^{2}\right) \mathrm{d} x \mathrm{~d} t+\frac{1}{\varepsilon} \mathbb{E} \int_{G} r_{\varepsilon}^{2}(0) \mathrm{d} x+\mathbb{E} \int_{Q} \mathrm{e}^{-2 \lambda \alpha_{\varepsilon}} \lambda^{-2} \gamma^{-2} R_{\varepsilon}^{2} \mathrm{~d} x \mathrm{~d} t \\
& \leq C \mathbb{E} \int_{Q} \theta^{2} \lambda^{3} \gamma^{3} h^{2} \mathrm{~d} x \mathrm{~d} t .
\end{aligned}
$$

Therefore, it is easy to check that there exists $(\widehat{g}, \widehat{r}, \widehat{R}) \in L_{\mathcal{F}}^{2}\left(0, T ; L^{2}\left(G_{1}\right)\right) \times L_{\mathcal{F}}^{2}\left(0, T ; H_{0}^{1}(G)\right) \times L_{\mathcal{F}}^{2}\left(0, T ; L^{2}(G)\right)$ such that as $\varepsilon \rightarrow 0$,

$$
\begin{aligned}
& g_{\varepsilon} \rightarrow \widehat{g} \quad \text { weakly in } L^{2}\left((0, T) \times \Omega ; L^{2}\left(G_{1}\right)\right) ; \\
& r_{\varepsilon} \rightarrow \widehat{r} \quad \text { weakly in } L^{2}\left((0, T) \times \Omega ; H_{0}^{1}(G)\right) ; \\
& R_{\varepsilon} \rightarrow \widehat{R} \quad \text { weakly in } L^{2}\left((0, T) \times \Omega ; L^{2}(G)\right) .
\end{aligned}
$$

Step 3. We conclude that $(\widehat{r}, \widehat{R})$ is the solution of $(2.1)$ associated to $\widehat{g}$. In fact, by the duality analysis as that in [27], it is easy to show that (2.1) has one and only one solution $(\widetilde{r}, \widetilde{R}) \in$ $\left(L_{\mathcal{F}}^{2}\left(\Omega ; C\left([0, T] ; L^{2}(G)\right)\right) \bigcap L_{\mathcal{F}}^{2}\left(0, T ; H_{0}^{1}(G)\right)\right) \times L_{\mathcal{F}}^{2}\left(0, T ; L^{2}(G)\right)$ associated to $\widehat{g} \in L_{\mathcal{F}}^{2}\left(0, T ; L^{2}\left(G_{1}\right)\right)$. Then, for any $f_{i} \in L_{\mathcal{F}}^{2}\left(0, T ; L^{2}(G)\right)(i=1,2)$, consider the following forward stochastic parabolic equation:

$$
\begin{cases}\mathrm{d} \phi-\sum_{i, j=1}^{n}\left(a^{i j} \phi_{x_{i}}\right)_{x_{j}} \mathrm{~d} t=f_{1} \mathrm{~d} t+f_{2} \mathrm{~d} w(t) & \text { in } Q, \\ \phi=0 & \text { on } \Sigma, \\ \phi(0)=0 & \text { in } G .\end{cases}
$$

By (2.1), (2.10) and Itô's formula, we see that

$$
\mathbb{E} \int_{Q}\left(\theta^{2} \lambda^{3} \gamma^{3} h+\chi_{G_{1}} \widehat{g}\right) \phi \mathrm{d} x \mathrm{~d} t+\mathbb{E} \int_{Q}\left(\widetilde{r} f_{1}+\widetilde{R} f_{2}\right) \mathrm{d} x \mathrm{~d} t=0
$$

and

$$
\mathbb{E} \int_{Q}\left(\theta^{2} \lambda^{3} \gamma^{3} h+\chi_{G_{1}} g_{\varepsilon}\right) \phi \mathrm{d} x \mathrm{~d} t+\mathbb{E} \int_{Q}\left(r_{\varepsilon} f_{1}+R_{\varepsilon} f_{2}\right) \mathrm{d} x \mathrm{~d} t=0
$$


which, together with (2.9), indicates that

$$
\mathbb{E} \int_{Q}\left(\theta^{2} \lambda^{3} \gamma^{3} h+\chi_{G_{1}} \widehat{g}\right) \phi \mathrm{d} x \mathrm{~d} t+\mathbb{E} \int_{Q}\left(\widehat{r} f_{1}+\widehat{R} f_{2}\right) \mathrm{d} x \mathrm{~d} t=0 .
$$

Therefore, by (2.11) and (2.12), it follows that $\widetilde{r}=\widehat{r}$ and $\widetilde{R}=\widehat{R}$ in $Q, \mathcal{P}$-a.s.

Moreover, by $(2.8)$, we get that $\widehat{r}(0)=0$ in $G, \mathcal{P}$-a.s. and $(2.2)$ holds.

Now, we prove the Carleman estimate (1.3) based on the null controllability result for the backward parabolic equation (2.1), by the duality technique again.

Proof of Theorem 1.1. For any $F_{i} \in L_{\mathcal{F}}^{2}\left(0, T ; L^{2}(G)\right)(i=1,2)$ and $h_{0} \in L^{2}\left(\Omega, \mathcal{F}_{0}, \mathcal{P} ; L^{2}(G)\right)$, let $h$ denote the corresponding solution of equation $(1.2)$ and $(\widehat{r}, \widehat{R})$ denote the solution of equation (2.1) associated to $\widehat{g}$, which satisfy all conditions mentioned in Proposition 2.2. Then, by Itô's formula, we see that

$$
\mathbb{E} \int_{Q} \theta^{2} \lambda^{3} \gamma^{3} h^{2} \mathrm{~d} x \mathrm{~d} t=-\mathbb{E} \int_{Q}\left(\chi_{G_{1}} \widehat{g} h+\widehat{r} F_{1}+\widehat{R} F_{2}\right) \mathrm{d} x \mathrm{~d} t .
$$

It follows that for sufficiently small $\rho>0$,

$$
\begin{aligned}
\mathbb{E} \int_{Q} \theta^{2} \lambda^{3} \gamma^{3} h^{2} \mathrm{~d} x \mathrm{~d} t \leq & \rho \mathbb{E} \int_{Q} \theta^{-2}\left(\lambda^{-3} \gamma^{-3} \widehat{g}^{2}+\widehat{r}^{2}+\lambda^{-2} \gamma^{-2} \widehat{R}^{2}\right) \mathrm{d} x \mathrm{~d} t \\
& +C(\rho)\left(\mathbb{E} \int_{0}^{T} \int_{G_{1}} \theta^{2} \lambda^{3} \gamma^{3} h^{2} \mathrm{~d} x \mathrm{~d} t+\mathbb{E} \int_{Q} \theta^{2} F_{1}^{2} \mathrm{~d} x \mathrm{~d} t+\mathbb{E} \int_{Q} \theta^{2} \lambda^{2} \gamma^{2} F_{2}^{2} \mathrm{~d} x \mathrm{~d} t\right) .
\end{aligned}
$$

By (2.2), this implies that

$$
\mathbb{E} \int_{Q} \theta^{2} \lambda^{3} \gamma^{3} h^{2} \mathrm{~d} x \mathrm{~d} t \leq C\left(\mathbb{E} \int_{0}^{T} \int_{G_{1}} \theta^{2} \lambda^{3} \gamma^{3} h^{2} \mathrm{~d} x \mathrm{~d} t+\mathbb{E} \int_{Q} \theta^{2} F_{1}^{2} \mathrm{~d} x \mathrm{~d} t+\mathbb{E} \int_{Q} \theta^{2} \lambda^{2} \gamma^{2} F_{2}^{2} \mathrm{~d} x \mathrm{~d} t\right) .
$$

On the other hand, notice that

$$
d\left(\theta^{2} \lambda \gamma h^{2}\right)=\left(\theta^{2} \lambda \gamma\right)_{t} h^{2} \mathrm{~d} t+2 \theta^{2} \lambda \gamma h\left[\sum_{i, j=1}^{n}\left(a^{i j} h_{x_{i}}\right)_{x_{j}} \mathrm{~d} t+F_{1} \mathrm{~d} t+F_{2} \mathrm{~d} w(t)\right]+\theta^{2} \lambda \gamma(\mathrm{d} h)^{2} .
$$

Therefore, by a simple calculation, we conclude that for sufficiently small $\rho>0$,

$$
\begin{aligned}
& 2 \mathbb{E} \int_{Q} \theta^{2} \lambda \gamma \sum_{i, j=1}^{n} a^{i j} h_{x_{i}} h_{x_{j}} \mathrm{~d} x \mathrm{~d} t \\
& =\mathbb{E} \int_{Q}\left[\left(\theta^{2} \lambda \gamma\right)_{t} h^{2}-2 \sum_{i, j=1}^{n} a^{i j}\left(\theta^{2} \lambda \gamma\right)_{x_{j}} h_{x_{i}} h+2 \theta^{2} \lambda \gamma h F_{1}+\theta^{2} \lambda \gamma F_{2}^{2}\right] \mathrm{d} x \mathrm{~d} t \\
& \leq C \mathbb{E} \int_{Q}\left(\theta^{2} \lambda^{2} \gamma^{3} h^{2}+\theta^{2} \lambda^{2} \gamma^{2}|\nabla h||h|+\theta^{2} \lambda \gamma\left|h F_{1}\right|+\theta^{2} \lambda \gamma F_{2}^{2}\right) \mathrm{d} x \mathrm{~d} t \\
& \leq \rho \mathbb{E} \int_{Q} \theta^{2} \lambda \gamma|\nabla h|^{2} \mathrm{~d} x \mathrm{~d} t+C(\rho)\left(\mathbb{E} \int_{Q} \theta^{2} \lambda^{3} \gamma^{3} h^{2} \mathrm{~d} x \mathrm{~d} t+\mathbb{E} \int_{Q} \theta^{2} F_{1}^{2} \mathrm{~d} x \mathrm{~d} t+\mathbb{E} \int_{Q} \theta^{2} \lambda \gamma F_{2}^{2} \mathrm{~d} x \mathrm{~d} t\right) .
\end{aligned}
$$


By (2.13), this implies that

$$
\mathbb{E} \int_{Q} \theta^{2} \lambda \gamma|\nabla h|^{2} \mathrm{~d} x \mathrm{~d} t \leq C\left(\mathbb{E} \int_{0}^{T} \int_{G_{1}} \theta^{2} \lambda^{3} \gamma^{3} h^{2} \mathrm{~d} x \mathrm{~d} t+\mathbb{E} \int_{Q} \theta^{2} F_{1}^{2} \mathrm{~d} x \mathrm{~d} t+\mathbb{E} \int_{Q} \theta^{2} \lambda^{2} \gamma^{2} F_{2}^{2} \mathrm{~d} x \mathrm{~d} t\right) .
$$

Combining (2.13) with (2.14), we arrive at the desired estimate (1.3).

Remark 2.3. As one of applications of the Carleman estimate (1.3), the known null controllability result for backward stochastic parabolic equations can be improved by relaxing the assumptions on some coefficients. Indeed, the assumptions on $a^{i j}, p$ in [23], Theorem 2.2 and on $c$ in [23], Theorem 2.4 can be relaxed as $a^{i j} \in$ $L_{\mathcal{F}}^{\infty}\left(\Omega ; C^{1}\left([0, T] ; W^{1, \infty}(G)\right)\right)$ and $p, c \in L_{\mathcal{F}}^{\infty}\left(0, T ; L^{\infty}(G)\right)$, respectively.

\section{Reduction of the InSENSITIZING CONTROL PROBLEMS}

As an application of the Carleman estimate (1.3), we shall prove the existence of insensitizing controls for the equation (1.5). As usual, we need to reduce this insensitivity problem to a partial controllability problem for some stochastic cascade parabolic system, as stated below.

Proposition 3.1. Given $\xi \in L_{\mathcal{F}}^{2}\left(0, T ; L^{2}(G)\right)$ and $y_{T} \in L^{2}\left(\Omega, \mathcal{F}_{T}, \mathcal{P} ; L^{2}(G)\right)$. Suppose that $(y, Y, z) \in$ $\left(L_{\mathcal{F}}^{2}\left(\Omega ; C\left([0, T] ; L^{2}(G)\right)\right) \bigcap L_{\mathcal{F}}^{2}\left(0, T ; H_{0}^{1}(G)\right)\right) \times L_{\mathcal{F}}^{2}\left(0, T ; L^{2}(G)\right) \times L_{\mathcal{F}}^{2}\left(\Omega ; C\left([0, T] ; H_{0}^{1}(G)\right)\right)$ is the solution of the following linear cascade stochastic parabolic system associated to a pair of controls $(u, v) \in L_{\mathcal{F}}^{2}\left(0, T ; L^{2}\left(G_{0}\right)\right) \times$ $L_{\mathcal{F}}^{2}\left(0, T ; L^{2}(G)\right)$ :

$$
\left\{\begin{array}{lr}
\mathrm{d} y+\Delta y \mathrm{~d} t=\left(\xi+\mu Y+\chi_{G_{0}} u\right) \mathrm{d} t+(v+Y) \mathrm{d} w(t) & \text { in } Q, \\
y=0 & \text { on } \Sigma, \\
y(T)=y_{T} & \text { in } G,
\end{array}\right.
$$

and

$$
\left\{\begin{array}{lr}
\mathrm{d} z-\Delta z \mathrm{~d} t=\chi_{\mathcal{O}_{1}} y \mathrm{~d} t+\left(-\mu z+\chi_{\mathcal{O}_{2}} Y\right) \mathrm{d} w(t) & \text { in } Q \\
z=0 & \text { on } \Sigma \\
z(0)=0 & \text { in } G
\end{array}\right.
$$

Then, $z(T)=0$ in $G, \mathcal{P}$-a.s. if and only if the insensitivity condition (1.7) holds for $(u, v)$.

Proof. For any $(u, v) \in L_{\mathcal{F}}^{2}\left(0, T ; L^{2}\left(G_{0}\right)\right) \times L_{\mathcal{F}}^{2}\left(0, T ; L^{2}(G)\right), \tau \in \mathbb{R}$ and $\widehat{y}_{T} \in L^{2}\left(\Omega, \mathcal{F}_{T}, \mathcal{P} ; L^{2}(G)\right)$

with $\left|\widehat{y}_{T}\right|_{L^{2}\left(\Omega, \mathcal{F}_{T}, \mathcal{P} ; L^{2}(G)\right)}=1$, we denote by $\left(y_{\tau}, Y_{\tau}\right)$ the solution of equation (1.5) associated to $\tau$ and $(u, v)$. Then, it follows that

$$
\left.\frac{\partial \Phi\left(y_{\tau}, Y_{\tau}\right)}{\partial \tau}\right|_{\tau=0}=\lim _{\tau \rightarrow 0} \frac{1}{2}\left[\mathbb{E} \int_{0}^{T} \int_{\mathcal{O}_{1}}\left(y_{\tau}+y\right) \frac{y_{\tau}-y}{\tau} \mathrm{d} x \mathrm{~d} t+\mathbb{E} \int_{0}^{T} \int_{\mathcal{O}_{2}}\left(Y_{\tau}+Y\right) \frac{Y_{\tau}-Y}{\tau} \mathrm{d} x \mathrm{~d} t\right],
$$

where $(y, Y)$ is the solution of equation (3.1) associated to $(u, v)$. Write $\bar{y}=\frac{y_{\tau}-y}{\tau}$ and $\bar{Y}=\frac{Y_{\tau}-Y}{\tau}$. Obviously, $(\bar{y}, \bar{Y})$ satisfies the following backward stochastic parabolic equation:

$$
\begin{cases}\mathrm{d} \bar{y}+\Delta \bar{y} \mathrm{~d} t=\mu \bar{Y} \mathrm{~d} t+\bar{Y} \mathrm{~d} w(t) & \text { in } Q, \\ \bar{y}=0 & \text { on } \Sigma, \\ \bar{y}(T)=\widehat{y}_{T} & \text { in } G .\end{cases}
$$


Then, by (3.2), (3.4) and Itô's formula, we see that

$$
\begin{aligned}
& \mathbb{E} \int_{G} \widehat{y}_{T} z(T) \mathrm{d} x=\mathbb{E} \int_{Q}\left[z(-\Delta \bar{y}+\mu \bar{Y})+\bar{y}\left(\Delta z+\chi_{\mathcal{O}_{1}} y\right)+\bar{Y}\left(-\mu z+\chi_{\mathcal{O}_{2}} Y\right)\right] \mathrm{d} x \mathrm{~d} t \\
& =\mathbb{E} \int_{0}^{T} \int_{\mathcal{O}_{1}} \bar{y} y \mathrm{~d} x \mathrm{~d} t+\mathbb{E} \int_{0}^{T} \int_{\mathcal{O}_{2}} \bar{Y} Y \mathrm{~d} x \mathrm{~d} t .
\end{aligned}
$$

Combining the above equality with (3.3), it follows that

$$
\left.\frac{\partial \Phi\left(y_{\tau}, Y_{\tau}\right)}{\partial \tau}\right|_{\tau=0}=\mathbb{E} \int_{G} \widehat{y}_{T} z(T) \mathrm{d} x, \forall \widehat{y}_{T} \in L^{2}\left(\Omega, \mathcal{F}_{T}, \mathcal{P} ; L^{2}(G)\right) \text { with }\left|\widehat{y}_{T}\right|_{L^{2}\left(\Omega, \mathcal{F}_{T}, \mathcal{P} ; L^{2}(G)\right)}=1 .
$$

Hence,

$$
\left.\frac{\partial \Phi\left(y_{\tau}, Y_{\tau}\right)}{\partial \tau}\right|_{\tau=0}=0 \quad \text { if and only if } \quad z(T)=0 \text { in } G, \mathcal{P} \text {-a.s. }
$$

This completes the proof of Proposition 3.1.

\section{Global Carleman estimate for a linear stochastic parabolic system}

As a preliminary to prove Theorem 1.6, the main goal of this section is to derive a global Carleman estimate for the following linear cascade stochastic parabolic system:

$$
\begin{cases}\mathrm{d} p+\Delta p \mathrm{~d} t=\mu P \mathrm{~d} t+P \mathrm{~d} w(t) & \text { in } Q, \\ \mathrm{~d} q-\Delta q \mathrm{~d} t=\chi_{\mathcal{O}_{1}} p \mathrm{~d} t+\left(\chi_{\mathcal{O}_{2}} P-\mu q\right) \mathrm{d} w(t) & \text { in } Q, \\ p=q=0 & \text { on } \Sigma, \\ p(T)=p_{T}, \quad q(0)=0 & \text { in } G,\end{cases}
$$

where $\mu \in L_{\mathcal{F}}^{\infty}\left(0, T ; L^{\infty}(G)\right)$ and $p_{T} \in L^{2}\left(\Omega, \mathcal{F}_{T}, \mathcal{P} ; L^{2}(G)\right)$.

In the sequel, we choose $k=2$ in the weighted functions $\gamma$ and $\theta$. Then, the main result of this section is the following global Carleman estimate for the system (4.1).

Proposition 4.1. If $\mathcal{O}_{2}=G$, there exists a positive constant $\widehat{\beta}$, depending only on $n, G, G_{0}, G_{1}$ and $T$ such that for any $\beta \geq \widehat{\beta}$, one can find two positive constants $\widehat{\lambda}=\widehat{\lambda}(\beta)$ and $C=C(\beta)$ so that solutions of the system (4.1) satisfy

$$
\begin{aligned}
& \mathbb{E} \int_{Q} \theta^{2}\left(p^{2}+\lambda^{-2} \gamma^{-2}|\nabla p|^{2}\right) \mathrm{d} x \mathrm{~d} t+\mathbb{E} \int_{Q} \theta^{2}\left(\lambda^{3} \gamma^{3} q^{2}+\lambda \gamma|\nabla q|^{2}\right) \mathrm{d} x \mathrm{~d} t \\
& \leq C\left[\mathbb{E} \int_{0}^{T} \int_{G_{0}} \theta^{2} \lambda^{4} \gamma^{4} q^{2} \mathrm{~d} x \mathrm{~d} t+\mathbb{E} \int_{Q} \theta^{2} \lambda^{2} \gamma^{2}(P-\mu q)^{2} \mathrm{~d} x \mathrm{~d} t\right],
\end{aligned}
$$

for any $p_{T} \in L^{2}\left(\Omega, \mathcal{F}_{T}, \mathcal{P} ; L^{2}(G)\right)$ and $\lambda \geq \hat{\lambda}$.

Before giving a proof of Proposition 4.1, consider the following backward stochastic parabolic equation:

$$
\begin{cases}\mathrm{d} h+\Delta h \mathrm{~d} t=F \mathrm{~d} t+H \mathrm{~d} w(t) & \text { in } Q \\ h=0 & \text { on } \Sigma, \\ h(T)=h_{T} & \text { in } G,\end{cases}
$$

where $F \in L_{\mathcal{F}}^{2}\left(0, T ; L^{2}(G)\right)$ and $h_{T} \in L^{2}\left(\Omega, \mathcal{F}_{T}, \mathcal{P} ; L^{2}(G)\right)$ are arbitrarily given. Then, we have the following known Carleman estimate for equation (4.2) (see [23], Thm. 6.1). 
Lemma 4.2. There exists a positive constant $\beta_{3}$, depending only on $n, G, G_{1}$ and $T$ such that for any $\beta \geq \beta_{3}$, one can find two positive constants $\lambda_{3}=\lambda_{3}(\beta)$ and $C=C(\beta)$, so that for any $F \in L_{\mathcal{F}}^{2}\left(0, T ; L^{2}(G)\right)$ and $h_{T} \in L^{2}\left(\Omega, \mathcal{F}_{T}, \mathcal{P} ; L^{2}(G)\right)$, any solution $(h, H) \in L_{\mathcal{F}}^{2}\left(0, T ; H_{0}^{1}(G)\right) \times L_{\mathcal{F}}^{2}\left(0, T ; L^{2}(G)\right)$ of equation (4.2) satisfies

$$
\mathbb{E} \int_{Q} \theta^{2}\left(\lambda^{3} \gamma^{3} h^{2}+\lambda \gamma|\nabla h|^{2}\right) \mathrm{d} x \mathrm{~d} t \leq C\left(\mathbb{E} \int_{0}^{T} \int_{G_{1}} \theta^{2} \lambda^{3} \gamma^{3} h^{2} \mathrm{~d} x \mathrm{~d} t+\mathbb{E} \int_{Q} \theta^{2} F^{2} \mathrm{~d} x \mathrm{~d} t+\mathbb{E} \int_{Q} \theta^{2} \lambda^{2} \gamma^{2} H^{2} \mathrm{~d} x \mathrm{~d} t\right),
$$

for any $\lambda \geq \lambda_{3}$.

Further, following the method used in [9], one has the following more general Carleman estimate for equation (4.2).

Lemma 4.3. There exists a positive constant $\beta_{3}$, depending only on $n, G, G_{1}$ and $T$ such that for any $\beta \geq \beta_{3}$, one can find two positive constants $\lambda_{3}=\lambda_{3}(\beta)$ and $C=C(\beta)$, so that for any $d \in \mathbb{R}, F \in L_{\mathcal{F}}^{2}\left(0, T ; L^{2}(G)\right)$ and $h_{T} \in L^{2}\left(\Omega, \mathcal{F}_{T}, \mathcal{P} ; L^{2}(G)\right)$, any solution $(h, H) \in L_{\mathcal{F}}^{2}\left(0, T ; H_{0}^{1}(G)\right) \times L_{\mathcal{F}}^{2}\left(0, T ; L^{2}(G)\right)$ of equation (4.2) satisfies

$$
\begin{aligned}
& \mathbb{E} \int_{Q} \theta^{2}\left(\lambda^{3} \gamma^{3+d} h^{2}+\lambda \gamma^{1+d}|\nabla h|^{2}\right) \mathrm{d} x \mathrm{~d} t \\
& \leq C\left(\mathbb{E} \int_{0}^{T} \int_{G_{1}} \theta^{2} \lambda^{3} \gamma^{3+d} h^{2} \mathrm{~d} x \mathrm{~d} t+\mathbb{E} \int_{Q} \theta^{2} \gamma^{d} F^{2} \mathrm{~d} x \mathrm{~d} t+\mathbb{E} \int_{Q} \theta^{2} \lambda^{2} \gamma^{2+d} H^{2} \mathrm{~d} x \mathrm{~d} t\right),
\end{aligned}
$$

for any $\lambda \geq \lambda_{3}$.

Sketch of the proof of Lemma 4.3. Write $\widehat{h}=\gamma^{\frac{d}{2}} h$ and $\widehat{H}=\gamma^{\frac{d}{2}} H$. Then, it is easy to check that

$$
d \widehat{h}+\Delta \widehat{h} \mathrm{~d} t=\left[\gamma^{\frac{d}{2}} F-d(T-2 t) \gamma^{\frac{1}{2}} \widehat{h}\right] \mathrm{d} t+\widehat{H} \mathrm{~d} w(t) \quad \text { in } Q .
$$

Applying Lemma 4.2 to $(\widehat{h}, \widehat{H})$, one can get the desired estimate in Lemma 4.3.

Now, we come back to the proof of Proposition 4.1.

Proof of Proposition 4.1. First, applying Lemma 4.3 and Theorem 1.1 to $p$ and $q$, respectively, we conclude that there exist two sufficiently large positive constants $\widehat{\beta}=\max \left(\beta_{1}, \beta_{3}\right)$ and $\widehat{\lambda}=\max \left(\lambda_{1}, \lambda_{3}\right)$ such that for $d=-3$, $\beta \geq \widehat{\beta}$ and $\lambda \geq \widehat{\lambda}$

$$
\mathbb{E} \int_{Q} \theta^{2}\left(p^{2}+\lambda^{-2} \gamma^{-2}|\nabla p|^{2}\right) \mathrm{d} x \mathrm{~d} t \leq C\left(\mathbb{E} \int_{0}^{T} \int_{G_{1}} \theta^{2} p^{2} \mathrm{~d} x \mathrm{~d} t+\mathbb{E} \int_{Q} \theta^{2} \lambda^{-1} \gamma^{-1} P^{2} \mathrm{~d} x \mathrm{~d} t\right),
$$

and

$$
\begin{aligned}
& \mathbb{E} \int_{Q} \theta^{2}\left(\lambda^{3} \gamma^{3} q^{2}+\lambda \gamma|\nabla q|^{2}\right) \mathrm{d} x \mathrm{~d} t \\
& \leq C\left[\mathbb{E} \int_{0}^{T} \int_{G_{1}} \theta^{2} \lambda^{3} \gamma^{3} q^{2} \mathrm{~d} x \mathrm{~d} t+\mathbb{E} \int_{Q} \theta^{2} \lambda^{2} \gamma^{2}(P-\mu q)^{2} \mathrm{~d} x \mathrm{~d} t+\mathbb{E} \int_{0}^{T} \int_{\mathcal{O}_{1}} \theta^{2} p^{2} \mathrm{~d} x \mathrm{~d} t\right] .
\end{aligned}
$$

Next, we estimate the last term in the right side of (4.4). Choose a nonnegative function $\zeta \in C_{0}^{\infty}\left(G_{0}\right)$ satisfying $\zeta=1$ in $G_{1}$. By (4.1), notice that

$$
\begin{aligned}
d\left(\zeta \theta^{2} p q\right)=\zeta\left(\theta^{2}\right)_{t} p q \mathrm{~d} t+\zeta \theta^{2} p \mathrm{~d} q+\zeta \theta^{2} q \mathrm{~d} p+\zeta \theta^{2} \mathrm{~d} p \mathrm{~d} q= & \zeta\left(\theta^{2}\right)_{t} p q \mathrm{~d} t+\zeta \theta^{2} p\left[\left(\Delta q+\chi_{\mathcal{O}_{1}} p\right) \mathrm{d} t+(P-\mu q) \mathrm{d} w(t)\right] \\
& +\zeta \theta^{2} q[(-\Delta p+\mu P) \mathrm{d} t+P \mathrm{~d} w(t)]+\zeta \theta^{2} \mathrm{~d} p \mathrm{~d} q
\end{aligned}
$$


Therefore, by integration by parts and a simple calculation, we get that for sufficiently small $\rho>0$,

$$
\begin{aligned}
\mathbb{E} \int_{0}^{T} \int_{G_{1}} \theta^{2} p^{2} \mathrm{~d} x \mathrm{~d} t & \leq-\mathbb{E} \int_{Q}\left[\zeta\left(\theta^{2}\right)_{t} p q+2 \nabla\left(\zeta \theta^{2}\right) \cdot \nabla p q+\Delta\left(\zeta \theta^{2}\right) p q+\zeta \theta^{2} P^{2}\right] \mathrm{d} x \mathrm{~d} t \\
& \leq C \mathbb{E} \int_{0}^{T} \int_{G_{0}}\left(\theta^{2} \lambda \gamma^{2}|p q|+\theta^{2} \lambda \gamma|\nabla p||q|+\theta^{2} \lambda^{2} \gamma^{2}|p q|\right) \mathrm{d} x \mathrm{~d} t \\
& \leq \rho \mathbb{E} \int_{Q} \theta^{2}\left(p^{2}+\lambda^{-2} \gamma^{-2}|\nabla p|^{2}\right) \mathrm{d} x \mathrm{~d} t+C(\rho) \mathbb{E} \int_{0}^{T} \int_{G_{0}} \theta^{2} \lambda^{4} \gamma^{4} q^{2} \mathrm{~d} x \mathrm{~d} t
\end{aligned}
$$

Combining the above inequality with (4.3), we find that

$$
\begin{aligned}
\mathbb{E} \int_{Q} \theta^{2}\left(p^{2}+\lambda^{-2} \gamma^{-2}|\nabla p|^{2}\right) \mathrm{d} x \mathrm{~d} t \leq & \frac{1}{2} \mathbb{E} \int_{Q} \theta^{2}\left(p^{2}+\lambda^{-2} \gamma^{-2}|\nabla p|^{2}\right) \mathrm{d} x \mathrm{~d} t \\
& +C \mathbb{E} \int_{0}^{T} \int_{G_{0}} \theta^{2} \lambda^{4} \gamma^{4} q^{2} \mathrm{~d} x \mathrm{~d} t+C \mathbb{E} \int_{Q} \theta^{2} \lambda^{-1} \gamma^{-1} P^{2} \mathrm{~d} x \mathrm{~d} t
\end{aligned}
$$

It follows that

$$
\mathbb{E} \int_{Q} \theta^{2}\left(p^{2}+\lambda^{-2} \gamma^{-2}|\nabla p|^{2}\right) \mathrm{d} x \mathrm{~d} t \leq C\left(\mathbb{E} \int_{0}^{T} \int_{G_{0}} \theta^{2} \lambda^{4} \gamma^{4} q^{2} \mathrm{~d} x \mathrm{~d} t+\mathbb{E} \int_{Q} \theta^{2} \lambda^{-1} \gamma^{-1} P^{2} \mathrm{~d} x \mathrm{~d} t\right) .
$$

This implies that

$$
\mathbb{E} \int_{0}^{T} \int_{\mathcal{O}_{1}} \theta^{2} p^{2} \mathrm{~d} x \mathrm{~d} t \leq C\left(\mathbb{E} \int_{0}^{T} \int_{G_{0}} \theta^{2} \lambda^{4} \gamma^{4} q^{2} \mathrm{~d} x \mathrm{~d} t+\mathbb{E} \int_{Q} \theta^{2} \lambda^{-1} \gamma^{-1} P^{2} \mathrm{~d} x \mathrm{~d} t\right) .
$$

Substituting (4.6) into (4.4), we see that

$$
\begin{aligned}
& \mathbb{E} \int_{Q} \theta^{2}\left(\lambda^{3} \gamma^{3} q^{2}+\lambda \gamma|\nabla q|^{2}\right) \mathrm{d} x \mathrm{~d} t \\
& \leq C\left[\mathbb{E} \int_{0}^{T} \int_{G_{0}} \theta^{2} \lambda^{4} \gamma^{4} q^{2} \mathrm{~d} x \mathrm{~d} t+\mathbb{E} \int_{Q} \theta^{2} \lambda^{2} \gamma^{2}(P-\mu q)^{2} \mathrm{~d} x \mathrm{~d} t+\mathbb{E} \int_{Q} \theta^{2} \lambda^{-1} \gamma^{-1} P^{2} \mathrm{~d} x \mathrm{~d} t\right] \\
& \leq C\left[\mathbb{E} \int_{0}^{T} \int_{G_{0}} \theta^{2} \lambda^{4} \gamma^{4} q^{2} \mathrm{~d} x \mathrm{~d} t+\mathbb{E} \int_{Q} \theta^{2} \lambda^{2} \gamma^{2}(P-\mu q)^{2} \mathrm{~d} x \mathrm{~d} t+\mathbb{E} \int_{Q} \theta^{2} \lambda^{-1} \gamma^{-1} q^{2} \mathrm{~d} x \mathrm{~d} t\right] .
\end{aligned}
$$

Hence, it follows that

$$
\mathbb{E} \int_{Q} \theta^{2}\left(\lambda^{3} \gamma^{3} q^{2}+\lambda \gamma|\nabla q|^{2}\right) \mathrm{d} x \mathrm{~d} t \leq C\left[\mathbb{E} \int_{0}^{T} \int_{G_{0}} \theta^{2} \lambda^{4} \gamma^{4} q^{2} \mathrm{~d} x \mathrm{~d} t+\mathbb{E} \int_{Q} \theta^{2} \lambda^{2} \gamma^{2}(P-\mu q)^{2} \mathrm{~d} x \mathrm{~d} t\right] .
$$

Finally, combining (4.5) and (4.7), we arrive at the desired estimate in Proposition 4.1.

Based on Proposition 4.1, one has the following observability estimate for equation (4.1), by borrowing some ideas from $[24,25]$.

Corollary 4.4. If $\mathcal{O}_{2}=G$, there exists a positive constant $M>0$, depending only on $n, T, G, G_{0}, \mathcal{O}_{1}$ and $|\mu|_{L_{\mathcal{F}}^{\infty}\left(0, T ; L^{\infty}(G)\right)}$ such that for any $p_{T} \in L^{2}\left(\Omega, \mathcal{F}_{T}, \mathcal{P} ; L^{2}(G)\right)$, any solution $(p, P, q)$ of equation (4.1) satisfies

$$
\mathbb{E} \int_{Q} \exp \left(\frac{-M}{(T-t)^{2}}\right) q^{2} \mathrm{~d} x \mathrm{~d} t \leq C\left[\mathbb{E} \int_{0}^{T} \int_{G_{0}} q^{2} \mathrm{~d} x \mathrm{~d} t+\mathbb{E} \int_{Q}(P-\mu q)^{2} \mathrm{~d} x \mathrm{~d} t\right] .
$$


Proof. First, we apply the usual energy estimate to the first two equations of (4.1). Notice that $d\left(p^{2}\right)=2 p \mathrm{~d} p+$ $(\mathrm{d} p)^{2}$. For any $t_{1}, t_{2} \in[0, T]$ with $t_{1}<t_{2}$, by Young's inequality, it is easy to see that

$$
\begin{aligned}
& \mathbb{E} \int_{G} p^{2}\left(t_{2}\right) \mathrm{d} x-\mathbb{E} \int_{G} p^{2}\left(t_{1}\right) \mathrm{d} x=\mathbb{E} \int_{t_{1}}^{t_{2}} \int_{G}\left[2 p(-\Delta p \mathrm{~d} t+\mu P \mathrm{~d} t)+P^{2} \mathrm{~d} t\right] \mathrm{d} x \\
& =2 \mathbb{E} \int_{t_{1}}^{t_{2}} \int_{G}|\nabla p|^{2} \mathrm{~d} x \mathrm{~d} t+2 \mathbb{E} \int_{t_{1}}^{t_{2}} \int_{G} \mu p P \mathrm{~d} x \mathrm{~d} t+\mathbb{E} \int_{t_{1}}^{t_{2}} \int_{G} P^{2} \mathrm{~d} x \mathrm{~d} t \\
& \geq-C \mathbb{E} \int_{t_{1}}^{t_{2}} \int_{G} p^{2} \mathrm{~d} x \mathrm{~d} t .
\end{aligned}
$$

Hence, in terms of the Gronwall inequality, it follows that

$$
\mathbb{E} \int_{G} p^{2}\left(t_{1}\right) \mathrm{d} x \leq C \mathbb{E} \int_{G} p^{2}\left(t_{2}\right) \mathrm{d} x, \quad \text { for any } 0 \leq t_{1}<t_{2} \leq T .
$$

This implies that

$$
\mathbb{E} \int_{G} p^{2}(t) \mathrm{d} x \leq C \mathbb{E} \int_{G} p^{2}\left(t+\frac{T}{4}\right) \mathrm{d} x, \quad \text { for any } t \in\left[0, \frac{T}{2}\right] .
$$

Integrating the above inequality on $\left(0, \frac{T}{2}\right)$, we obtain that

$$
\mathbb{E} \int_{0}^{\frac{T}{2}} \int_{G} p^{2} \mathrm{~d} x \mathrm{~d} t \leq C \mathbb{E} \int_{\frac{T}{4}}^{\frac{3 T}{4}} \int_{G} p^{2} \mathrm{~d} x \mathrm{~d} t .
$$

On the other hand, note that $d\left(q^{2}\right)=2 q \mathrm{~d} q+(\mathrm{d} q)^{2}$. For any $t_{1}, t_{2} \in[0, T]$ with $t_{1}<t_{2}$, by Young's inequality, it is easy to see that

$$
\begin{aligned}
& \mathbb{E} \int_{G} q^{2}\left(t_{2}\right) \mathrm{d} x-\mathbb{E} \int_{G} q^{2}\left(t_{1}\right) \mathrm{d} x=\mathbb{E} \int_{t_{1}}^{t_{2}} \int_{G}\left[2 q\left(\Delta q \mathrm{~d} t+\chi_{\mathcal{O}_{1}} p \mathrm{~d} t\right)+(P-\mu q)^{2} \mathrm{~d} t\right] \mathrm{d} x \\
& =-2 \mathbb{E} \int_{t_{1}}^{t_{2}} \int_{G}|\nabla q|^{2} \mathrm{~d} x \mathrm{~d} t+2 \mathbb{E} \int_{t_{1}}^{t_{2}} \int_{\mathcal{O}_{1}} p q \mathrm{~d} x \mathrm{~d} t+\mathbb{E} \int_{t_{1}}^{t_{2}} \int_{G}(P-\mu q)^{2} \mathrm{~d} x \mathrm{~d} t \\
& \leq \mathbb{E} \int_{t_{1}}^{t_{2}} \int_{G} q^{2} \mathrm{~d} x \mathrm{~d} t+\mathbb{E} \int_{t_{1}}^{t_{2}} \int_{G} p^{2} \mathrm{~d} x \mathrm{~d} t+\mathbb{E} \int_{t_{1}}^{t_{2}} \int_{G}(P-\mu q)^{2} \mathrm{~d} x \mathrm{~d} t .
\end{aligned}
$$

By the Gronwall inequality, we get that

$$
\mathbb{E} \int_{G} q^{2}(t) \mathrm{d} x \leq C\left[\mathbb{E} \int_{0}^{t} \int_{G} p^{2} \mathrm{~d} x \mathrm{~d} t+\mathbb{E} \int_{0}^{t} \int_{G}(P-\mu q)^{2} \mathrm{~d} x \mathrm{~d} t\right], \quad \text { for any } t \in[0, T] .
$$

Integrating the above inequality on $\left(0, \frac{T}{2}\right)$, it follows that

$$
\mathbb{E} \int_{0}^{\frac{T}{2}} \int_{G} q^{2} \mathrm{~d} x \mathrm{~d} t \leq C\left[\mathbb{E} \int_{0}^{\frac{T}{2}} \int_{G} p^{2} \mathrm{~d} x \mathrm{~d} t+\mathbb{E} \int_{0}^{\frac{T}{2}} \int_{G}(P-\mu q)^{2} \mathrm{~d} x \mathrm{~d} t\right] .
$$

Next, it is easy to check that there exists a positive constant $M>0$, depending only on $n, T, G, G_{0}, G_{1}, \mathcal{O}_{1}$ and $|\mu|_{L_{\mathcal{F}}^{\infty}\left(0, T ; L^{\infty}(G)\right)}$, such that for two fixed constants $\beta$ and $\lambda$ satisfying the conditions mentioned in Proposition 4.1,

$$
\exp \left(\frac{-M}{(T-t)^{2}}\right) \leq C \exp \left(2 \lambda \frac{\mathrm{e}^{\beta \psi}-\mathrm{e}^{2 \beta|\psi|_{C(\bar{G})}}}{t^{2}(T-t)^{2}}\right) t^{-6}(T-t)^{-6}, \quad \text { in }\left(\frac{T}{2}, T\right) \times G .
$$


Hence, by Proposition 4.1, we get that

$$
\mathbb{E} \int_{\frac{T}{2}}^{T} \int_{G} \exp \left(\frac{-M}{(T-t)^{2}}\right) q^{2} \mathrm{~d} x \mathrm{~d} t \leq C \mathbb{E} \int_{Q} \theta^{2} \gamma^{3} q^{2} \mathrm{~d} x \mathrm{~d} t \leq C\left[\mathbb{E} \int_{0}^{T} \int_{G_{0}} q^{2} \mathrm{~d} x \mathrm{~d} t+\mathbb{E} \int_{Q}(P-\mu q)^{2} \mathrm{~d} x \mathrm{~d} t\right] .
$$

Moreover, by (4.10), (4.9) and Proposition 4.1, we see that

$$
\begin{aligned}
\mathbb{E} \int_{0}^{\frac{T}{2}} \int_{G} \exp \left(\frac{-M}{(T-t)^{2}}\right) q^{2} \mathrm{~d} x \mathrm{~d} t & \leq \mathbb{E} \int_{0}^{\frac{T}{2}} \int_{G} q^{2} \mathrm{~d} x \mathrm{~d} t \\
& \leq C\left[\mathbb{E} \int_{0}^{\frac{T}{2}} \int_{G} p^{2} \mathrm{~d} x \mathrm{~d} t+\mathbb{E} \int_{0}^{\frac{T}{2}} \int_{G}(P-\mu q)^{2} \mathrm{~d} x \mathrm{~d} t\right] \\
& \leq C\left[\mathbb{E} \int_{\frac{T}{4}}^{\frac{3 T}{4}} \int_{G} p^{2} \mathrm{~d} x \mathrm{~d} t+\mathbb{E} \int_{0}^{\frac{T}{2}} \int_{G}(P-\mu q)^{2} \mathrm{~d} x \mathrm{~d} t\right] \\
& \leq C\left[\mathbb{E} \int_{\frac{T}{4}}^{\frac{3 T}{4}} \int_{G} \theta^{2} p^{2} \mathrm{~d} x \mathrm{~d} t+\mathbb{E} \int_{0}^{\frac{T}{2}} \int_{G}(P-\mu q)^{2} \mathrm{~d} x \mathrm{~d} t\right] \\
& \leq C\left[\mathbb{E} \int_{0}^{T} \int_{G_{0}} q^{2} \mathrm{~d} x \mathrm{~d} t+\mathbb{E} \int_{Q}(P-\mu q)^{2} \mathrm{~d} x \mathrm{~d} t\right] .
\end{aligned}
$$

Combining (4.11) with (4.12), we get the desired inequality (4.8).

Remark 4.5. If $\mathcal{O}_{2} \varsubsetneqq G$, in order to get the desired controllability result for the system (3.1)-(3.2), one has to prove that there exists a positive constant $M>0$, depending only on $n, T, G, G_{0}, \mathcal{O}_{1}, \mathcal{O}_{2}$ and $\mu$ such that for any $p_{T} \in L^{2}\left(\Omega, \mathcal{F}_{T}, \mathcal{P} ; L^{2}(G)\right)$, any solution $(p, P, q)$ of equation (4.1) satisfies the following estimate:

$$
\mathbb{E} \int_{Q} \exp \left(\frac{-M}{(T-t)^{2}}\right) q^{2} \mathrm{~d} x \mathrm{~d} t \leq C\left[\mathbb{E} \int_{0}^{T} \int_{G_{0}} q^{2} \mathrm{~d} x \mathrm{~d} t+\mathbb{E} \int_{Q}\left(\chi_{\mathcal{O}_{2}} P-\mu q\right)^{2} \mathrm{~d} x \mathrm{~d} t\right] .
$$

However, this observability inequality seems very difficult.

\section{Proof of the insensitivity ReSUlt}

In the following, we prove the existence of insensitizing controls for (1.5).

Proof of Theorem 1.6. First, we introduce the following linear subspace of $L_{\mathcal{F}}^{2}\left(0, T ; L^{2}\left(G_{0}\right)\right) \times L_{\mathcal{F}}^{2}\left(0, T ; L^{2}(G)\right)$ :

$$
L=\left\{\left(\left.q\right|_{\Omega \times(0, T) \times G_{0}}, P-\mu q\right) \mid(p, P, q) \text { solves (4.1) with some } p_{T} \in L^{2}\left(\Omega, \mathcal{F}_{T}, \mathcal{P} ; L^{2}(G)\right)\right\}
$$

and define a linear functional on $L$ as follows:

$$
\mathcal{L}\left(\left.q\right|_{\Omega \times(0, T) \times G_{0}}, P-\mu q\right)=-\mathbb{E} \int_{Q} q \xi \mathrm{d} x \mathrm{~d} t .
$$

By means of (4.8), for any $\xi \in L_{\mathcal{F}}^{2}\left(0, T ; L^{2}(G)\right)$ satisfying $\left|\exp \left(\frac{M}{(T-t)^{2}}\right) \xi\right|_{L_{\mathcal{F}}^{2}\left(0, T ; L^{2}(G)\right)}<\infty$, we see that $\mathcal{L}$ is a bounded linear functional on $L$. Then, by the Hahn-Banach theorem, $\mathcal{L}$ can be extended to a bounded linear functional on $L_{\mathcal{F}}^{2}\left(0, T ; L^{2}\left(G_{0}\right)\right) \times L_{\mathcal{F}}^{2}\left(0, T ; L^{2}(G)\right)$. For simplicity, we use the same notation for this extension. Therefore, one can find a pair of random fields $(u, v) \in L_{\mathcal{F}}^{2}\left(0, T ; L^{2}\left(G_{0}\right)\right) \times L_{\mathcal{F}}^{2}\left(0, T ; L^{2}(G)\right)$ such that

$$
\mathbb{E} \int_{0}^{T} \int_{G_{0}} u q \mathrm{~d} x \mathrm{~d} t+\mathbb{E} \int_{Q} v(P-\mu q) \mathrm{d} x \mathrm{~d} t=-\mathbb{E} \int_{Q} q \xi \mathrm{d} x \mathrm{~d} t
$$


Moreover, there exists a positive constant $C$, depending only on $n, T, G, G_{0}, \mathcal{O}_{1}$ and $|\mu|_{L_{\mathcal{F}}^{\infty}\left(0, T ; L^{\infty}(G)\right)}$ such that

$$
|u|_{L_{\mathcal{F}}^{2}\left(0, T ; L^{2}\left(G_{0}\right)\right)}+|v|_{L_{\mathcal{F}}^{2}\left(0, T ; L^{2}(G)\right)} \leq C\left|\exp \left(\frac{M}{(T-t)^{2}}\right) \xi\right|_{L_{\mathcal{F}}^{2}\left(0, T ; L^{2}(G)\right)} .
$$

Next, we prove that the pair of random fields $(u, v)$ is the desired control. Indeed, for any $p_{T} \in$ $L^{2}\left(\Omega, \mathcal{F}_{T}, \mathcal{P} ; L^{2}(G)\right)$, by the system (3.1)-(3.2), equation (4.1) and Itô's formula, we see

$$
\begin{aligned}
& \mathbb{E} \int_{G} p_{T} z(T) \mathrm{d} x=\mathbb{E} \int_{Q}(p \mathrm{~d} z+z \mathrm{~d} p+\mathrm{d} p \mathrm{~d} z-y \mathrm{~d} q-q \mathrm{~d} y-\mathrm{d} y \mathrm{~d} q) \mathrm{d} x \\
& =-\left[\mathbb{E} \int_{Q} q \xi \mathrm{d} x \mathrm{~d} t+\mathbb{E} \int_{0}^{T} \int_{G_{0}} u q \mathrm{~d} x \mathrm{~d} t+\mathbb{E} \int_{Q} v(P-\mu q) \mathrm{d} x \mathrm{~d} t\right] .
\end{aligned}
$$

Combining (5.1) with the above equality, we obtain

$$
\mathbb{E} \int_{G} p_{T} z(T) \mathrm{d} x=0, \quad \text { for any } p_{T} \in L^{2}\left(\Omega, \mathcal{F}_{T}, \mathcal{P} ; L^{2}(G)\right)
$$

Hence, we get that $z(T)=0$ in $G$, P-a.s. By Proposition 3.1, we complete the proof of Theorem 1.6.

Remark 5.1. As pointed out in Proposition 3.1, the key in the proof of Theorem 1.6 is to show a controllability result for the coupled linear stochastic parabolic system (3.1)-(3.2). In the last decades, there are many works addressing the controllability of deterministic parabolic equations/systems (e.g. $[1,5,6,26,29]$ and the rich references therein). However, as far as we know, very little is known about the controllability of stochastic parabolic equations. We refer to $[16,23]$ for some known results in this respect. Note however that the desired controllability result in this paper involves solving a controllability problem for some coupled system governed by a backward stochastic parabolic equation and a forward one. Therefore, it is technically more difficult to treat this sort of controllability problem than the case of single stochastic parabolic equations.

Remark 5.2. Compared to the insensitivity problems for forward stochastic parabolic equations, there exists an essential new difficulty in the study of the same problem but for backward stochastic parabolic equations. Indeed, the key observability estimate in [25] was established by means of the known Carleman estimates (in [23]) for forward and backward stochastic parabolic equations. However, when we study the insensitivity control problem for the backward stochastic parabolic equation (1.5), the known Carleman estimates are not enough to establish the desired observability estimate for the involved cascade system (4.1). In fact, the first equation in this cascade system is backward, and therefore it has two state variables $p$ and $P$. Further, the diffusion term of the second forward equation in (4.1) contains $P$. Consequently, if trying to employ simply the known Carleman estimate (in [23]) for forward stochastic parabolic equations, one would need to establish a suitable estimate for $\nabla P$, but, this sort of estimates seem to be unavailable. In order to overcome this difficulty, we use the improved Carleman estimate (1.3) to establish the desired controllability result for the coupled parabolic system (3.1)-(3.2).

Acknowledgements. The author thanks Professor Xu Zhang (Sichuan University) for his valuable suggestions to improve this paper.

\section{REFERENCES}

[1] F. Ammar-Khodja, A. Benabdallah, M. González-Burgos and L. de Teresa, Recent results on the controllability of linear coupled parabolic problems: A survey. Math. Control Relat. Fields 1 (2011) 267-306.

[2] O. Bodart, M. González-Burgos and R. Pérez-Garcia, A local result on insensitizing controls for a semilinear heat equation with nonlinear boundary Fourier conditions. SIAM J. Control Optim. 43 (2004) 955-969. 
[3] A.-P. Calderón, Uniqueness in the Cauchy problem for partial differential equations. Amer. J. Math. 80 (1958) $16-36$.

[4] T. Carleman, Sur un problème d'unicité pur les systèmes d'équations aux dérivées partielles à deux variables indépendantes. Ark. Mat. Astr. Fys. 26 (1939) 1-9.

[5] X. Fu, A weighted identity for partial differential operator of second order and its applications. C.R. Math. Acad. Sci. Paris 342 (2006) 579-584.

[6] A.V. Fursikov and O. Yu. Imanuvilov, Controllability of Evolution Equations. In vol. 34, Lect. Notes Ser. Seoul National University, Seoul, Korea (1996).

[7] L. Hörmander, Linear Partial Differential Operators, in vol. 116. Die Grundlehren der mathematischen Wissenschaften. Academic Press, New York (1963).

[8] O. Yu. Imanuvilov and J.P. Puel, Global Carleman estimates for weak solutions of elliptic nonhomogeneous Dirichlet problems. Int. Math. Res. Not. 16 (2003) 883-913.

[9] O. Yu. Imanuvilov and M. Yamamoto, Carleman inequalities for parabolic equations in Sobolev spaces of negative order and exact controllability for semilinear parabolic equations. Publ. Res. Inst. Math. Sci. 39 (2003) 227-274.

[10] N.V. Krylov, A $W_{2}^{n}$-theory of the Dirichlet problem for SPDEs in general smooth domains. Prob. Theory Related Fields 98 (1994) 389-421.

[11] X. Li and J. Yong, Optimal Control Theory for Infinite-Dimensional Systems. Birkhäuser Boston, Inc., Boston (1995).

[12] J.-L. Lions, Quelques notions dans l'analyse et le contrôle de systèmes à données incomplètes, Proc. of the XIth Congress on Differential Equations and Applications/First Congress on Appl. Math. Univ. Málaga, Málaga (1990) 43-54.

[13] J.-L. Lions, Optimal Control of Systems Governed by Partial Differential Equations, vol. 170. Springer-Verlag, New York-Berlin (1971).

[14] X. Liu, Insensitizing controls for a class of quasilinear parabolic equations. J. Differ. Eqs. 253 (2012) $1287-1316$.

[15] X. Liu and X. Zhang, Local controllability of multidimensional quasi-linear parabolic equations. SIAM J. Control Optim. 50 (2012) 2046-2064.

[16] Q. Lü, Some results on the controllability of forward stochastic heat equations with control on the drift. J. Funct. Anal. 260 (2011) 832-851.

[17] Q. Lü, Carleman estimate for stochastic parabolic equations and inverse stochastic parabolic problems. Inverse Problems 28 (2012) 045008

[18] Q. Lü and X. Zhang, Carleman estimates for parabolic operators with discontinuous and anisotropic diffusion coefficients, an elementary approach. In preparation.

[19] Q. Lü and X. Zhang, General Pontryagin-type stochastic maximum principle and backward stochastic evolution equations in infinite dimensions, arXiv:1204.3275.

[20] J. Ma and J. Yong, Adapted solution of a degenerate backward SPDE, with applications. Stochastic Process. Appl. 70 (1997) 59-84.

[21] E. Pardoux, Stochastic partial differential equations and filtering of diffusion processes. Stochastic 3 (1979) $127-167$.

[22] J.-C. Saut and B. Scheurer, Unique continuation for some evolution equations. J. Differ. Eqs. 66 (1987) 118-139.

[23] S. Tang and X. Zhang, Null controllability for forward and backward stochastic parabolic equations. SIAM J. Control Optim. 48 (2009) 2191-2216.

[24] L. de Teresa, Insensitizing controls for a semilinear heat equation. Commun. Partial Differ. Eqs. 25 (2000) 39-72.

[25] Y. Yan and F. Sun, Insensitizing controls for a forward stochastic heat equation. J. Math. Anal. Appl. 384 (2011) 138-150.

[26] X. Zhang, A unified controllability/observability theory for some stochastic and deterministic partial differential equations. Proc. of the Int. Congress of Math., Vol. IV. Hyderabad, India (2010) 3008-3034.

[27] X. Zhou, A duality analysis on stochastic partial differential equations. J. Funct. Anal. 103 (1992) $275-293$.

[28] X. Zhou, On the necessary conditions of optimal controls for stochastic partial differential equations. SIAM J. Control Optim. 31 (1993) 1462-1478.

[29] E. Zuazua, Controllability and Observability of Partial Differential Equations: Some results and open problems. Handbook of Differential Equations: Evol. Differ. Eqs., vol. 3. Elsevier Science (2006) 527-621.

[30] C. Zuily, Uniqueness and Non-Uniqueness in the Cauchy Problem. Birkhäuser Verlag, Boston-Basel-Stuttgart (1983). 